\title{
SPECTRAL INEQUALITIES FOR SCHRÖDINGER OPERATORS WITH SURFACE POTENTIALS
}

\author{
RUPERT L. FRANK AND ARI LAPTEV \\ Dedicated to M. Sh. Birman on the occasion of his 80th birthday
}

\begin{abstract}
We prove sharp Lieb-Thirring inequalities for Schrödinger operators with potentials supported on a hyperplane and we show how these estimates are related to LiebThirring inequalities for relativistic Schrödinger operators.
\end{abstract}

\section{INTRODUCTION}

The Cwikel-Lieb-Rozenblum and the Lieb-Thirring inequalities estimate the number and moments of eigenvalues of Schrödinger operators $-\Delta-V$ in $L_{2}\left(\mathbb{R}^{N}\right)$ in terms of an integral of the potential $V$. They state that the bound

$$
\operatorname{tr}(-\Delta-V)_{-}^{\gamma} \leq L_{\gamma, N} \int_{\mathbb{R}^{N}} V(x)_{+}^{\gamma+N / 2} d x
$$

holds with a constant $L_{\gamma, N}$ independent of $V$ iff $\gamma \geq 1 / 2$ for $N=1, \gamma>0$ for $N=2$ and $\gamma \geq 0$ for $N \geq 3$. Here and below $t_{ \pm}:=\max \{0, \pm t\}$ denotes the positive and negative part of a real number, a real-valued function or a self-adjoint operator $t$. In particular, the problem of finding the optimal value of the constant $L_{\gamma, N}$ has attracted a lot of attention recently. We refer to the review articles [H2, LW2] for background information, references and applications of (1.1).

The purpose of the present paper is twofold. First, we would like to find an analog of inequality (1.1) for Schrödinger operators with singular potentials $V(x)=v\left(x_{1}, \ldots, x_{d}\right) \delta\left(x_{N}\right)$, $d:=N-1$, supported on a hyperplane. It turns out that such an inequality is indeed valid, provided the integral on the right hand side of (1.1) is replaced by

$$
\int_{\mathbb{R}^{d}} v\left(x_{1}, \ldots, x_{d}\right)_{+}^{2 \gamma+d} d x_{1} \ldots d x_{d}
$$

We determine the complete range of $\gamma$ 's for which the resulting inequality holds. Moreover, we find the sharp values of the constants for $\gamma \geq 3 / 2$ by using the method of 'lifting with respect to the dimension'. This provides yet another example of the power and flexibility of this method, which was used by Laptev and Weidl [LW1] to obtain the sharp constants in (1.1) for $\gamma \geq 3 / 2$.

The second purpose of this paper is to point out a relation between the Schrödinger operator $-\Delta-v\left(x_{1}, \ldots, x_{d}\right) \delta\left(x_{N}\right)$ in $L_{2}\left(\mathbb{R}^{N}\right)$ and the relativistic Schrödinger operator $\sqrt{-\Delta}-$ $v\left(x_{1}, \ldots, x_{d}\right)$ in $L_{2}\left(\mathbb{R}^{d}\right)$. Note that the space dimension $d=N-1$ of the relativistic operator differs from that of the non-relativistic operator. The basic idea is to relate eigenfunctions of the Schrödinger operator with singular potential to eigenfunctions of a non-linear eigenvalue

(C) 2007 by the authors. This paper may be reproduced, in its entirety, for non-commercial purposes. 
problem involving the relativistic Schrödinger operator. This construction is essentially the Poisson extension and is implicit in several earlier works, e.g., in [CL, FS, CS]. In our context the connection between the two operators becomes useful when combined with a monotonicity argument in the spirit of the Birman-Schwinger principle. It allows us both to prove the singular analog of inequality (1.1) and to (slightly) improve upon the known constants in Lieb-Thirring inequalities for relativistic Schrödinger operators.

\section{SCHRÖDINGER OPERATORS WITH SURFACE POTENTIALS}

2.1. Main results. In this section we consider the operator

$$
H(v) u=-\Delta u \quad \text { in } \mathbb{R}_{+}^{d+1}:=\left\{(x, y): x \in \mathbb{R}^{d}, y>0\right\}
$$

together with boundary conditions of the third type

$$
\frac{\partial u}{\partial \nu}-v u=0 \quad \text { on } \mathbb{R}^{d} \times\{0\} .
$$

Here $\partial / \partial \nu=-\partial / \partial y$ denotes the (exterior) normal derivative and $v$ a real-valued function on $\mathbb{R}^{d}$. If $v$ is form-compact with respect to $\sqrt{-\Delta}$ in $L_{2}\left(\mathbb{R}^{d}\right)$, then $H(v)$ can be defined as a self-adjoint operator in $L_{2}\left(\mathbb{R}_{+}^{d+1}\right)$ by means of the quadratic form

$$
\iint_{\mathbb{R}_{+}^{d+1}}|\nabla u|^{2} d x d y-\int_{\mathbb{R}^{d}} v(x)|u(x, 0)|^{2} d x, \quad u \in H^{1}\left(\mathbb{R}_{+}^{d+1}\right) .
$$

The negative spectrum of $H(v)$ consists of eigenvalues of finite multiplicities. We shall prove

Theorem 2.1 (Lieb-Thirring inequalities for surface potentials). The inequality

$$
\operatorname{tr}[H(v)]_{-}^{\gamma} \leq S_{\gamma, d} \int_{\mathbb{R}^{d}} v(x)_{+}^{2 \gamma+d} d x
$$

holds for all $0 \leq v \in L_{2 \gamma+d}\left(\mathbb{R}^{d}\right)$ iff

$$
\gamma>0 \quad \text { if } d=1, \quad \text { and } \quad \gamma \geq 0 \quad \text { if } d \geq 2 .
$$

Inequality (2.4) reflects the correct order of growth in the strong coupling limit, as can be seen from the Weyl-type asymptotics

$$
\lim _{\alpha \rightarrow \infty} \alpha^{-2 \gamma-d} \operatorname{tr}[H(\alpha v)]_{-}^{\gamma}=L_{\gamma, d}^{\mathrm{cl}} \int_{\mathbb{R}^{d}} v(x)_{+}^{2 \gamma+d} d x
$$

with

$$
L_{\gamma, d}^{\mathrm{cl}}:=2^{-d} \pi^{-d / 2} \frac{\Gamma(\gamma+1)}{\Gamma(\gamma+d / 2+1)} .
$$

Since relation (2.6) is not completely standard we comment on its proof in Remark 3.7 below.

Our second result concerns the constants in the bounds of Theorem 2.1, Denoting by $S_{\gamma, d}$ the sharp constant in (2.4) we infer from (2.6) that

$$
S_{\gamma, d} \geq L_{\gamma, d}^{\mathrm{cl}}
$$

We shall prove that for sufficiently large values of $\gamma$ one actually has equality.

Theorem 2.2 (Sharp constants). Let $d \geq 1$ and $\gamma \geq 3 / 2$. Then the sharp constant in (2.4) is $S_{\gamma, d}=L_{\gamma, d}^{\mathrm{cl}}$. 
We prove this theorem in Subsection 2.2, Besides the sharp constants for $\gamma \geq 3 / 2$ our method yields explicit and tight bounds for $S_{\gamma, d}$ for arbitrary $\gamma$. In particular, we prove

$$
\begin{aligned}
S_{\gamma, d} \leq \frac{\pi}{\sqrt{3}} L_{\gamma, d}^{\mathrm{cl}} & \text { if } 1 \leq \gamma<3 / 2 \text { and } d \geq 1, \\
S_{\gamma, 1} \leq 2 L_{\gamma, 1}^{\mathrm{cl}} & \text { if } 1 / 2 \leq \gamma<1 \text { and } d=1, \\
S_{\gamma, d} \leq \frac{2 \pi}{\sqrt{3}} L_{\gamma, d}^{\mathrm{cl}} & \text { if } 1 / 2 \leq \gamma<1 \text { and } d \geq 2,
\end{aligned}
$$

see Remark 2.4. Moreover, the constants in the estimates for the number of negative eigenvalues satisfy

$$
\begin{aligned}
S_{0,2} \leq 6.04 L_{0,2}^{\mathrm{cl}} & \text { if } \gamma=0 \text { and } d=2, \\
S_{0,3} \leq 6.07 L_{0,3}^{\mathrm{cl}} & \text { if } \gamma=0 \text { and } d=3, \\
S_{0, d} \leq 10.332 L_{0, d}^{\mathrm{cl}} & \text { if } \gamma=0 \text { and } d \geq 4,
\end{aligned}
$$

see Remark 2.4 and Subsection 3.3. The upper bounds (2.10), (2.11) can be supplemented by the lower bounds

$$
\begin{array}{ll}
S_{0,2} \geq 4 L_{0,2}^{\mathrm{cl}} & \text { if } \gamma=0 \text { and } d=2, \\
S_{0,3} \geq 3 L_{0,3}^{\mathrm{cl}} & \text { if } \gamma=0 \text { and } d=3,
\end{array}
$$

see Subsection 3.3. In particular, for $d=2$ the upper bound (2.10) is off by at most a factor 1.51 .

2.2. Lifting with respect to dimension. In this subsection we use an argument in the spirit of Laptev and Weidl [LW1] to prove

Theorem 2.3. Let $d \geq 1, \gamma \geq 3 / 2$ and $\tau \geq 0$. Then

$$
\operatorname{tr}[H(v)+\tau]_{-}^{\gamma} \leq L_{\gamma, d}^{\mathrm{cl}} \int_{\mathbb{R}^{d}}\left(v(x)_{+}^{2}-\tau\right)_{+}^{\gamma+d / 2} d x
$$

with $L_{\gamma, d}^{\mathrm{cl}}$ defined in (2.7).

Choosing $\tau=0$ and recalling (2.6) we obtain Theorem 2.2 ,

Proof. We shall prove Theorem 2.3 by induction over $d$. It is convenient to reflect the dependence on $d$ in the notation of the quadratic form, so we write

$$
h_{d}(v)[u]:=\iint_{\mathbb{R}_{+}^{d+1}}|\nabla u|^{2} d x d y-\int_{\mathbb{R}^{d}} v(x)|u(x, 0)|^{2} d x d y
$$

and $H_{d}(v)$ for the associated operator. Note that this operator is also well-defined for $d=0$ and $v$ a non-negative real number. Indeed, in this case one has $H_{0}(v) u=-u^{\prime \prime}$ and $u^{\prime}(0)=-v u(0)$ for $u \in \operatorname{dom} H_{0}(v)$, and one easily finds that $H_{0}(v)$ has one negative eigenvalue, namely $-v_{+}^{2}$. Hence

$$
\operatorname{tr}_{L_{2}\left(\mathbb{R}_{+}\right)}\left[H_{0}(v)+\tau\right]_{-}^{\gamma}=\left(v_{+}^{2}-\tau\right)_{+}^{\gamma},
$$

which is the analog of (2.15) for $d=0$ and all $\gamma \geq 0$. 
Now we fix $d \geq 1$ and assume that the assertion is already proved for all smaller dimensions. We write $x=\left(x_{1}, x^{\prime}\right)$ with $x_{1} \in \mathbb{R}, x^{\prime} \in \mathbb{R}^{d-1}$ and note that

$$
H_{d}(v)+\tau \geq-\frac{d^{2}}{d x_{1}^{2}} \otimes 1_{L_{2}\left(\mathbb{R}_{+}^{d}\right)}-\left[H_{d-1}\left(v\left(x_{1}, \cdot\right)\right)+\tau\right]_{-}
$$

with the identification $L_{2}\left(\mathbb{R}_{+}^{d+1}\right)=L_{2}(\mathbb{R}) \otimes L_{2}\left(\mathbb{R}_{+}^{d}\right)$. Hence the variational principle and the operator-valued Lieb-Thirring inequality from [LW1] yields for all $\gamma \geq 3 / 2$

$$
\operatorname{tr}_{L_{2}\left(\mathbb{R}_{+}^{d+1}\right)}\left[H_{d}(v)+\tau\right]_{-}^{\gamma} \leq L_{\gamma, 1}^{\mathrm{cl}} \int_{-\infty}^{\infty} \operatorname{tr}_{L_{2}\left(\mathbb{R}_{+}^{d}\right)}\left[H_{d-1}\left(v\left(x_{1}, \cdot\right)\right)+\tau\right]_{-}^{\gamma+1 / 2} d x_{1} .
$$

By induction hypothesis, the right hand side is bounded from above by

$$
L_{\gamma, 1}^{\mathrm{cl}} L_{\gamma+1 / 2, d-1}^{\mathrm{cl}} \int_{-\infty}^{\infty}\left(\int_{\mathbb{R}^{d-1}}\left(v\left(x_{1}, x^{\prime}\right)_{+}^{2}-\tau\right)_{+}^{\gamma+d / 2} d x^{\prime}\right) d x_{1}=L_{\gamma, d}^{\mathrm{cl}} \int_{\mathbb{R}^{d}}\left(v(x)_{+}^{2}-\tau\right)_{+}^{\gamma+d / 2} d x,
$$

which establishes the assertion for dimension $d$ and completes the proof of Theorem 2.3 ,

Remark 2.4. The above approach can be used to prove inequality (2.4) for $\gamma \geq 1 / 2$ and to obtain bounds (2.9) for the sharp constants $S_{\gamma, d}$. Indeed, according to [HLW] and [DLL] the operator-valued inequality (2.16) holds with an additional factor of $\pi / \sqrt{3}$ on the right hand side if $\gamma \geq 1$, with an additional factor of 2 if $1 / 2 \leq \gamma<1$ and $d=1$ and with an additional factor of $2 \pi / \sqrt{3}$ if $\gamma \geq 1 / 2$ and $d \geq 2$.

Similarly, one can use the operator-valued inequality from [FLS2] (see also [H1]) to prove (2.4) for $\gamma \geq 0$ and $d \geq 3$ and to obtain (2.12). Extending the original proof of Lieb and Thirring [LT] to the operator-valued case would yield (2.4) for $\gamma>0$ and $d=2$. However, we do not know how to prove (2.4) with the operator-valued approach for $0<\gamma<1 / 2$ if $d=1$ and for $\gamma=0$ if $d=2$. We give a proof based on a different idea in Subsection 3.3 below.

\subsection{Additional remarks.}

2.3.1. Magnetic fields. Let $A \in L_{2, \text { loc }}\left(\overline{\mathbb{R}_{+}^{d+1}}, \mathbb{R}^{d+1}\right)$ and let the operator $H(A, v)$ be defined through the closure of the quadratic form

$$
\iint_{\mathbb{R}_{+}^{d+1}}|(-i \nabla-A) u|^{2} d x d y-\int_{\mathbb{R}^{d}} v(x)|u(x, 0)|^{2} d x, \quad u \in C_{0}^{\infty}\left(\overline{\mathbb{R}_{+}^{d+1}}\right) .
$$

By a similar argument as in [LW1] one can prove that Theorem 2.3 remains true, with the same constant, if $H(v)$ is replaced by $H(A, v)$. More generally, all the inequalities sketched in Remark 2.4 remain true. The argument behind Theorem 2.1, however, allows only $A$ which are independent of $y$ and orthogonal to the $y$-direction, see Remark 3.5. To obtain the analog of (2.4) for the complete range of $\gamma$ 's given in (2.5), one can rely upon an abstract operator-theoretic argument, see $[\mathbb{R}]$ for $\gamma=0$ and $[\mathbf{F}]$ for $\gamma>0$.

2.3.2. Leaky graph Hamiltonians. In the previous subsections we studied eigenvalues of the Laplacian $H(v)$ on the halfspace with a perturbation by boundary conditions. This problem is essentially equivalent to the study of eigenvalues of the Schrödinger operator

$$
\tilde{H}(v)=-\Delta-v(x) \delta(y)
$$


in the whole space $\mathbb{R}^{d+1}$ with a potential supported on a hyperplane. The precise definition of the operator $\tilde{H}(v)$ in $L_{2}\left(\mathbb{R}^{d+1}\right)$ is given via the quadratic form

$$
\iint_{\mathbb{R}^{d+1}}|\nabla u|^{2} d x d y-\int_{\mathbb{R}^{d}} v(x)|u(x, 0)|^{2} d x, \quad u \in H^{1}\left(\mathbb{R}^{d+1}\right) .
$$

The decomposition of a function into an even and an odd part with respect to the variable $y$ induces an orthogonal decomposition of the space $L_{2}\left(\mathbb{R}^{d+1}\right)$, which reduces the operator $\tilde{H}(v)$. The part of $\tilde{H}(v)$ on odd functions is unitarily equivalent to the Dirichlet Laplacian on $\mathbb{R}_{+}^{d+1}$, whereas the part on even functions is unitarily equivalent to $H\left(\frac{1}{2} v\right)$. Hence

$$
\operatorname{tr}[\tilde{H}(v)]_{-}^{\gamma}=\operatorname{tr}\left[H\left(\frac{1}{2} v\right)\right]_{-}^{\gamma}
$$

and we obtain immediately the analogs of Theorems 2.1 and 2.2 .

Theorem 2.5. The inequality

$$
\operatorname{tr}[\tilde{H}(v)]_{-}^{\gamma} \leq \tilde{S}_{\gamma, d} \int_{\mathbb{R}^{d}} v(x)_{+}^{2 \gamma+d} d x
$$

holds for all $0 \leq v \in L_{2 \gamma+d}\left(\mathbb{R}^{d}\right)$ iff

$$
\gamma>0 \quad \text { if } d=1, \quad \text { and } \quad \gamma \geq 0 \quad \text { if } d \geq 2 .
$$

Theorem 2.6. Let $d \geq 1$ and $\gamma \geq 3 / 2$. Then the sharp constant in (2.17) is $\tilde{S}_{\gamma, d}=$ $2^{-2 \gamma-d} L_{\gamma, d}^{\mathrm{cl}}$.

2.3.3. Complex-valued surface potentials. In applications one often encounters the boundary value problem (2.1), (2.2) with a complex-valued function $v$, which leads to non-real eigenvalues. If $v$ is sufficiently regular, the quadratic form (2.3) generates an $m$-sectorial operator which we continue to denote by $H(v)$. We denote by $\lambda_{j}(v), j=1,2, \ldots$, the (at most countably many) eigenvalues of $H(v)$ in the cut plane $\mathbb{C} \backslash[0, \infty)$, repeated according to their algebraic multiplicities. Following the approach suggested in [FLLS] one obtains

Theorem 2.7. Let $d \geq 1$ and $\gamma \geq 1$.

(1) For eigenvalues with negative real part

$$
\sum_{j: \operatorname{Re} \lambda_{j}(v)<0}\left(-\operatorname{Re} \lambda_{j}(v)\right)^{\gamma} \leq S_{\gamma, d} \int_{\mathbb{R}^{d}}(\operatorname{Re} v(x))_{-}^{2 \gamma+d} d x .
$$

(2) If $\kappa>0$, then for eigenvalues outside the cone $\{|\operatorname{Im} z|<\kappa \operatorname{Re} z\}$

$$
\sum_{j:\left|\operatorname{Im} \lambda_{j}(v)\right| \geq \kappa \operatorname{Re} \lambda_{j}(v)}\left|\lambda_{j}(v)\right|^{\gamma} \leq 2^{1+\gamma+d / 2}\left(1+\frac{2}{\kappa}\right)^{2 \gamma+d} S_{\gamma, d} \int_{\mathbb{R}^{d}}|v(x)|^{2 \gamma+d} d x .
$$

Here $S_{\gamma, d}$ is the constant from (2.4).

2.3.4. Waveguides. Let $\omega \subset \mathbb{R}^{d}$ be a domain of finite measure and put $\Omega:=\omega \times \mathbb{R}_{+}$and $\Gamma:=(\partial \omega) \times \mathbb{R}_{+}$. The quadratic form (2.3), restricted to $\left\{u \in H^{1}(\Omega): u=0\right.$ on $\left.\Gamma\right\}$, defines a self-adjoint operator $H_{\omega}(v)$ in $L_{2}(\Omega)$, which corresponds to Dirichlet boundary conditions on $\Gamma$ and boundary conditions of the third type on $\omega \times\{0\}$. By the variational principle Theorem 2.1 implies that

$$
\operatorname{tr}\left[H_{\omega}(v)\right]_{-}^{\gamma} \leq S_{\gamma, d} \int_{\omega} v(x)_{-}^{2 \gamma+d} d x
$$


for $\gamma>0$ if $d=1$ and $\gamma \geq 0$ if $d \geq 2$ with the constant $S_{\gamma, d}$ from (2.4). In particular, $S_{\gamma, d}=L_{\gamma, d}^{\mathrm{cl}}$ for $\gamma \geq 3 / 2$. We now show that in the special case where $v \equiv v_{0}$ is a constant, the estimate with the semi-classical constant holds already for $\gamma \geq 1$.

Theorem 2.8. Let $\omega \subset \mathbb{R}^{d}$ be a domain of finite measure and $v \equiv v_{0}>0$ a constant. Then for any $\gamma \geq 1$

$$
\operatorname{tr}\left[H_{\omega}(v)\right]_{-}^{\gamma} \leq L_{\gamma, d}^{\mathrm{cl}}|\omega| v_{0}^{2 \gamma+d} .
$$

Proof. By separation of variables one has

$$
\operatorname{tr}\left[H_{\omega}(v)\right]_{-}^{\gamma}=\operatorname{tr}\left[-\Delta_{\omega}^{D}-v_{0}^{2}\right]_{-}^{\gamma},
$$

where $-\Delta_{\omega}^{D}$ denotes the Dirichlet Laplacian on $\omega$. Therefore the assertion follows from the Berezin-Li-Yau inequality; see [B, LY] and also [L].

The same argument shows that if $\omega$ is tiling (in particular, any interval $\omega$ if $d=1$ ), then (2.19) holds for all $\gamma \geq 0$; see $[\mathrm{P}$.

\section{Relativistic Schrödinger OpERATORS}

3.1. Statement of the results. In this section we derive a connection between Schrödinger operators $H(v)$ with surface potential in $L_{2}\left(\mathbb{R}_{+}^{d+1}\right)$ and relativistic Schrödinger operators $\sqrt{-\Delta}-v$ in $L_{2}\left(\mathbb{R}^{d}\right)$. We begin by recalling Lieb-Thirring and Cwikel-Lieb-Rozenblum inequalities for the latter operator.

Proposition 3.1. The inequality

$$
\operatorname{tr}[\sqrt{-\Delta}-v]_{-}^{\gamma} \leq D_{\gamma, d} \int_{\mathbb{R}^{d}} v(x)_{+}^{\gamma+d} d x
$$

holds for all $0 \leq v \in L_{\gamma+d}\left(\mathbb{R}^{d}\right)$ iff

$$
\gamma>0 \quad \text { if } d=1, \quad \text { and } \quad \gamma \geq 0 \quad \text { if } d \geq 2 .
$$

This result is due to Daubechies [D]. The fact that the inequality is not valid for $\gamma=0$ if $d=1$ follows from the fact that $\sqrt{-\Delta}-v$ has a negative eigenvalue for any non-trivial $v \geq 0$ if $d=1$. This can be proved as in [S, Prop. 7.4].

The Weyl-type asymptotics in the relativistic case read

$$
\lim _{\alpha \rightarrow \infty} \alpha^{-\gamma-d} \operatorname{tr}[\sqrt{-\Delta}-\alpha v]_{-}^{\gamma}=D_{\gamma, d}^{\mathrm{cl}} \int_{\mathbb{R}^{d}} v(x)_{+}^{\gamma+d} d x
$$

with

$$
D_{\gamma, d}^{\mathrm{cl}}:=2^{-d} \pi^{-d / 2} \frac{\Gamma(\gamma+1) \Gamma(d+1)}{\Gamma(\gamma+d+1) \Gamma(d / 2+1)} .
$$

We denote by $D_{\gamma, d}$ the sharp constant in (3.1). In the case $d=3$, the bound

$$
D_{\gamma, 3} \leq 6.08 D_{\gamma, 3}^{\mathrm{cl}}, \quad \gamma \geq 0,
$$

is contained in [D]. Similarly one proves that for $d=2$

$$
D_{\gamma, 2} \leq 6.04 D_{\gamma, 2}^{\mathrm{cl}}, \quad \gamma \geq 0 .
$$

We are now in position to state a result which connects relativistic Schrödinger operator and non-relativistic Schrödinger operators with surface potentials. As usual, we denote by $N(-\tau, T)$ the number of eigenvalues, counting multiplicities, less than $-\tau$ of a self-adjoint, lower semi-bounded operator $T$, and write $N(T):=N(0, T)$. 
Theorem 3.2. Assume that $v$ is form-compact with respect to $\sqrt{-\Delta}$. Then for any $\tau \geq 0$ one has

$$
N(-\tau, H(v))=N(\sqrt{-\Delta+\tau}-v) .
$$

Moreover, for any $\gamma>0$ and $0<\rho<1$ one has

$$
\operatorname{tr}[\sqrt{-\Delta}-v]_{-}^{\gamma} \leq \operatorname{tr}[H(v)]_{-}^{\gamma / 2} \leq\left(\frac{\rho}{\sqrt{1-\rho^{2}}}\right)^{\gamma} \operatorname{tr}\left[\sqrt{-\Delta}-\rho^{-1} v\right]_{-}^{\gamma} .
$$

We shall prove this in Subsection 3.2 below, as well as the following

Corollary 3.3. The sharp constants in (2.4) and (3.1) coincide for $\gamma=0$ and $d \geq 2$,

$$
S_{0, d}=D_{0, d},
$$

and satisfy for any $\gamma>0$ and $d \geq 1$

$$
\frac{\gamma^{\gamma / 2} d^{d / 2}}{(\gamma+d)^{(\gamma+d) / 2}} S_{\gamma / 2, d} \leq D_{\gamma, d} \leq S_{\gamma / 2, d}
$$

We shall use Theorem 3.2 in two directions. In Subsection 3.3 we shall use the known Lieb-Thirring inequalities in the relativistic case to derive the Lieb-Thirring inequalities for surface potentials. In Subsection 3.4 we shall use the estimates on the constants $S_{\gamma, d}$ for surface potentials to improve upon the estimates (3.5) and (3.6) in the relativistic case. We also discuss the connection of our inequality with an inequality by Birman, Koplienko and Solomyak.

3.2. Duality. The following lemma characterizes the negative eigenvalues of the operator $H(v)$ as the values $-\tau$ for which 0 is an eigenvalue of the operator $\sqrt{-\Delta+\tau}-v$.

Lemma 3.4. Assume that $v$ is form-compact with respect to $\sqrt{-\Delta}$ and let $\tau>0$.

(1) Let $f \in \operatorname{ker}(\sqrt{-\Delta+\tau}-v)$ and define $u(x, y):=(\exp (-y \sqrt{-\Delta+\tau}) f)(x)$. Then $u \in \operatorname{ker}(H(v)+\tau)$ and $u(x, 0)=f(x)$.

(2) Let $u \in \operatorname{ker}(H(v)+\tau)$ and define $f(x):=u(x, 0)$. Then $f \in \operatorname{ker}(\sqrt{-\Delta+\tau}-v)$ and $u(x, y)=(\exp (-y \sqrt{-\Delta+\tau}) f)(x)$.

The proof of this lemma is straightforward and will be omitted (see [FS] for a similar argument). Using a modification of the Birman-Schwinger principle we now give the

Proof of Theorem 3.2. Since the eigenvalues of the operators $\sqrt{-\Delta+t}-v$ are increasing with respect to $t$, one has for any fixed $\tau \geq 0$

$$
N(\sqrt{-\Delta+\tau}-v)=\#_{\mathrm{m}}\{t>\tau: 0 \text { is an eigenvalue of } \sqrt{-\Delta+t}-v\} .
$$

Here $\#_{\mathrm{m}}\{\ldots\}$ means that the cardinality of $\{\ldots\}$ is determined according to multiplicities. By Lemma 3.4, the right hand side coincides with

$$
\#_{\mathrm{m}}\{t>\tau:-t \text { is an eigenvalue of } H(v)\}=N(-\tau, H(v)),
$$

as claimed.

To prove (3.8) we note that by the previous argument

$$
\operatorname{tr}[H(v)]_{-}^{\gamma / 2}=\frac{\gamma}{2} \int_{0}^{\infty} N(-\tau, H(v)) \tau^{\gamma / 2-1} d \tau=\frac{\gamma}{2} \int_{0}^{\infty} N(\sqrt{-\Delta+\tau}-v) \tau^{\gamma / 2-1} d \tau .
$$


The elementary inequalities

$$
\rho \sqrt{\lambda}+\sqrt{1-\rho^{2}} \sqrt{\tau} \leq \sqrt{\lambda+\tau} \leq \sqrt{\lambda}+\sqrt{\tau}, \quad \lambda, \tau>0,0<\rho<1,
$$

imply

$$
N(-\sqrt{\tau}, \sqrt{-\Delta}-v) \leq N(\sqrt{-\Delta+\tau}-v) \leq N\left(-\sqrt{1-\rho^{2}} \sqrt{\tau}, \rho \sqrt{-\Delta}-v\right) .
$$

Plugging this into (3.11) we obtain (3.8).

Proof of Corollary 3.3. Equality (3.9) as well as the second inequality in (3.10) follow immediately from equality (3.7) and the first inequality in (3.8). To prove the first inequality in (3.10) we combine Daubechies' inequality (3.1) with the second inequality in (3.8) to get

$$
\operatorname{tr}[H(v)]_{-}^{\gamma / 2} \leq D_{\gamma, d}\left(\frac{\rho}{\sqrt{1-\rho^{2}}}\right)^{\gamma} \rho^{-\gamma-d} \int_{\mathbb{R}^{d}} v(x)_{+}^{\gamma+d} d x .
$$

The assertion follows by optimizing over $0<\rho<1$.

Remark 3.5. The material in this subsection, except for the proof of the second part of Corollary 3.3, is of abstract nature. If $A$ is a non-negative operator in a Hilbert space $\mathfrak{H}$ and $B$ is a self-adjoint operator which is relatively form-compact with respect to $A$, define the operator $H$ in $L_{2}\left(\mathbb{R}_{+}, \mathfrak{H}\right)$ by the quadratic form

$$
\int_{0}^{\infty}\left(\left\|F^{\prime}(y)\right\|_{\mathfrak{H}}^{2}+\|A F(y)\|_{\mathfrak{H}}^{2}\right) d y-b[F(0)]
$$

for $F \in H^{1}\left(\mathbb{R}_{+}, \mathfrak{H}\right) \cap L_{2}\left(\mathbb{R}_{+}, \operatorname{dom} A\right)$. Here $b$ is the quadratic form of $B$. Then the argument of this subsection yields

$$
N(-\tau, H)=N\left(\sqrt{A^{2}+\tau}-B\right) .
$$

As an application of this generalization one can extend Theorem 3.2 to relativistic Schrödinger operators with magnetic field or to relativistic Schrödinger operators with a Hardy weight subtracted (see [FLS1]).

3.3. Proof of Theorem 2.1. Theorem 2.1 is an immediate consequence of Proposition 3.1 and Theorem 3.2 .

Moreover, Daubechies' bounds (3.6), (3.5) yield the upper bounds (2.10), (2.11) for the sharp constants $S_{0, d}$. Similarly, the lower bounds (2.13), (2.14) follow from

$$
D_{0, d} \geq \frac{2^{d-1}}{(d-1)^{d}} \Gamma(d+1) D_{0, d}^{\mathrm{cl}}, \quad d \geq 2 .
$$

(Note that this is only useful for $d \leq 7$, since otherwise the factor on the right hand side is smaller than one and the bound $D_{0, d} \geq D_{0, d}^{\mathrm{cl}}$ follows from (3.3).) The lower bound (3.12) can be seen as follows. The definition of $D_{0, d}$ implies that if $\int v^{d} d x<D_{0, d}^{-1}$, then $\sqrt{-\Delta}-v$ is a non-negative operator. Hence

$$
\int_{\mathbb{R}^{d}} v|u|^{2} d x \leq \int_{\mathbb{R}^{d}}\left|(-\Delta)^{1 / 4} u\right|^{2} d x
$$

for all $u \in H^{1 / 2}\left(\mathbb{R}^{d}\right)$. Choosing $v=\alpha|u|^{2 /(d-1)}$ with $\alpha$ such that $\alpha^{d} \int|u|^{2 d /(d-1)} d x=$ $\left(D_{0, d}+\varepsilon\right)^{-1}$ and letting $\varepsilon$ tend to zero, we find

$$
D_{0, d}^{-1 / d}\left(\int_{\mathbb{R}^{d}}|u|^{2 d /(d-1)} d x\right)^{(d-1) / d} \leq \int_{\mathbb{R}^{d}}\left|(-\Delta)^{1 / 4} u\right|^{2} d x
$$


for all $u \in H^{1 / 2}\left(\mathbb{R}^{d}\right)$. Hence $D_{0, d}^{-1 / d}$ is not larger than the constant in the sharp Sobolev inequality

$$
S_{d}^{\prime}\|u\|_{2 d /(d-1)}^{2} \leq\left\|(-\Delta)^{1 / 4} u\right\|^{2}, \quad S_{d}^{\prime}:=\frac{d-1}{2} 2^{1 / d} \pi^{(d+1) / 2 d} \Gamma\left(\frac{d+1}{2}\right)^{-1 / d} .
$$

see [LL, Thm. 8.4]. Recalling definition (3.4) of $D_{\gamma, d}^{\mathrm{cl}}$ we arrive at (3.12).

Remark 3.6. Instead of using the 'relativistic' Sobolev inequality (3.13) to prove (3.12) we could have used a similar argument based on the sharp Sobolev trace inequality

$$
S_{d}^{\prime}\left(\int_{\mathbb{R}^{d}}|u|^{2 d /(d-1)} d x\right)^{(d-1) / d} \leq \iint_{\mathbb{R}_{+}^{d+1}}|\nabla u|^{2} d x d y,
$$

to directly prove (2.13), (2.14). The constant $S_{d}^{\prime}$ in (3.14) is the same as in (3.13), see [E]. Indeed, an argument similar to our Lemma 3.4 was used in [CL to derive (3.14) from (3.13).

Remark 3.7. Weyl-type asymptotics (2.6) can be proved by a bracketing argument, dividing $\mathbb{R}_{+}^{d+1}$ into domains $Q \times \mathbb{R}_{+}$with $Q \subset \mathbb{R}^{d}$ a small cube. An alternative proof can be based on Theorem 3.2. Indeed, for $\gamma=0$ the asymptotics (2.6) follow immediately from Theorem 3.2 and (3.3) (which is valid for all smooth $v$ ). If $\gamma>0$ we write as in (3.11)

$$
\operatorname{tr}[H(\alpha v)]_{-}^{\gamma}=\gamma \int_{0}^{\infty} N(-\tau, H(\alpha v)) \tau^{\gamma-1} d \tau=\gamma \int_{0}^{\infty} N(\sqrt{-\Delta+\tau}-\alpha v) \tau^{\gamma-1} d \tau .
$$

For smooth $v$ one can justify that this is asymptotically equal as $\alpha \rightarrow \infty$ to

$$
\begin{aligned}
\gamma \int_{0}^{\infty} & \iint_{\left\{(x, \xi) \in \mathbb{R}^{d} \times \mathbb{R}^{d}: \sqrt{|\xi|^{2}+\tau}-\alpha v(x)<0\right\}} \frac{d x d \xi}{(2 \pi)^{d}} \tau^{\gamma-1} d \tau \\
= & \gamma L_{0, d}^{\mathrm{cl}} \int_{0}^{\infty} \int_{\mathbb{R}^{d}}\left((\alpha v(x))_{+}^{2}-\tau\right)_{+}^{d / 2} d x \tau^{\gamma-1} d \tau \\
= & \alpha^{2 \gamma+d} L_{\gamma, d}^{\mathrm{cl}} \int_{\mathbb{R}^{d}} v(x)_{+}^{2 \gamma+d} d x .
\end{aligned}
$$

This concludes the sketch of (2.6). We note that by a standard argument based on Theorem 2.1, the asymptotics (2.6) extend to all $v$ for which the right hand side is finite if $\gamma>0$ and $d=1$ or if $\gamma \geq 0$ and $d \geq 2$.

3.4. Relation with the BKS inequality. In this subsection we shall use Theorem 3.2 to improve upon known constants for relativistic Schrödinger operators and discuss its relation with an inequality by Birman, Koplienko and Solomyak. We begin with a result about massive relativistic Schrödinger operators.

Remark 3.8. Let $d \geq 3$ and $m \geq 0$. Then

$$
N\left(\sqrt{-\Delta+m^{2}}-m-v\right) \leq 10.332 D_{0, d}^{\mathrm{cl}} \int_{\mathbb{R}^{d}}\left((v(x)+m)_{+}^{2}-m^{2}\right)_{+}^{d / 2} d x .
$$

This improves upon Daubechies' bound [D] who obtains (3.15) with constant $14.14 D_{0,3}^{\mathrm{cl}}$ for $d=3$. To prove (3.15) we combine Theorem 3.2 and Remark 2.3 to get

$N\left(\sqrt{-\Delta+m^{2}}-m-v\right)=N\left(-m^{2}, H(v+m)\right) \leq 10.332 L_{0, d}^{\mathrm{cl}} \int_{\mathbb{R}^{d}}\left((v(x)+m)_{+}^{2}-m^{2}\right)_{+}^{d / 2} d x$, and recall that $L_{0, d}^{\mathrm{cl}}=D_{0, d}^{\mathrm{cl}}$. 
We return again to the massless case $m=0$.

Remark 3.9. The sharp constants in (3.1) satisfy

$$
\begin{array}{cl}
D_{\gamma, 2} \leq \sqrt{3} \pi D_{\gamma, 2}^{\mathrm{cl}} & \text { if } 2 \leq \gamma<3 \text { and } d=2, \\
D_{\gamma, 2} \leq 4 D_{\gamma, 2}^{\mathrm{cl}} & \text { if } \gamma \geq 3 \text { and } d=2, \\
D_{\gamma, 3} \leq \frac{15 \pi}{8} D_{\gamma, 3}^{\mathrm{cl}} & \text { if } \gamma \geq 3 \text { and } d=3 .
\end{array}
$$

This improves upon (3.6) and (3.5). To prove the first inequality in (3.16) we combine (3.10) with (2.9) to get

$$
D_{2,2} \leq \frac{\pi}{\sqrt{3}} L_{1,2}^{\mathrm{cl}}=\sqrt{3} \pi D_{2,2}^{\mathrm{cl}} .
$$

By the argument of Aizenman and Lieb [AL], this implies $D_{\gamma, 2} \leq \sqrt{3} \pi D_{\gamma, 2}^{\mathrm{cl}}$ for all $\gamma \geq 2$. The other bounds in (3.16) are proved similarly.

In conclusion we would like to recall a result by Birman, Koplienko and Solomyak.

Proposition 3.10. Let $0<s<1, \gamma \geq 1$ and $A, B$ non-negative operators such that $\operatorname{tr}(A-B)_{+}^{s \gamma}<\infty$. Then

$$
\operatorname{tr}\left(A^{s}-B^{s}\right)_{+}^{\gamma} \leq \operatorname{tr}(A-B)_{+}^{s \gamma}
$$

In $[\mathrm{BKS}]$ this is proved under the additional assumption $A \geq B$, but, as observed in [LSS], this assumption can be removed in view of the operator inequality

$$
A^{s}-B^{s} \leq\left(B+(A-B)_{+}\right)^{s}-B^{s} .
$$

Moreover, [LSS] contains an elementary proof of (3.17) in the case $\gamma=1$. We deduce from (3.17) and (1.1) that

$$
\operatorname{tr}(\sqrt{-\Delta}-v)_{-}^{\gamma} \leq \operatorname{tr}\left(-\Delta-v_{+}^{2}\right)_{-}^{\gamma / 2} \leq L_{\gamma / 2, d} \int_{\mathbb{R}^{d}} v(x)_{+}^{\gamma+d} d x, \quad \gamma \geq 1
$$

Since the best known bounds on the constants $L_{\gamma / 2, d}$ for $\gamma \geq 1$ coincide with those for $S_{\gamma / 2, d}$, Proposition 3.10yields for $\gamma \geq 1$ the same bounds on $D_{\gamma, d}$ as our Theorem 3.2. In particular, Remark 3.9 can also be derived via (3.18). In contrast, the $\gamma=0$ result of Remark 3.8 cannot be deduced via (3.18). It is interesting, in our opinion, to understand whether there is a deeper connection between the $s=1 / 2$ case of Proposition 3.10 and Theorem 3.2 .

Acknowledgments. This work has been supported by DAAD grant D/06/49117 (R. F.).

\section{REFERENCES}

[AL] M. Aizenman, E. Lieb, On semiclassical bounds for eigenvalues of Schrödinger operators. Phys. Lett. A 66 (1978), no. 6, 427-429.

[B] F.A. Berezin, Covariant and contravariant symbols of operators [Russian]. Math. USSR Izv. 6 (1972), 1117-1151.

[BKS] M. S. Birman, L. S. Koplienko, M. Z. Solomyak, Estimates for the spectrum of the difference between fraction powers of two self-adjoint operators. Soviet Math. (Iz. VUZ) 19 (1975), no. 3, 1-6.

[CL] E. Carlen, M. Loss, Competing symmetries of some functionals arising in mathematical physics. In: Stochastic processes, physics and geometry (Ascona and Locarno, 1988), 277-288, World Sci. Publ., Teaneck, NJ, 1990.

[CS] L. Caffarelli, L. Silvestre, An extension problem related to the fractional Laplacian. Comm. Partial Differential Equations 32 (2007), no. 8, 1245-1260. 
[D] I. Daubechies, An uncertainty principle for fermions with generalized kinetic energy. Comm. Math. Phys. 90 (1983), no. 4, 511-520.

[DLL] J. Dolbeault, A. Laptev, M. Loss, Lieb-Thirring inequalities with improved constants. J. Eur. Math. Soc., to appear.

[E] J. Escobar, Sharp constant in a Sobolev trace inequality. Indiana Univ. Math. J. 37 (1988), $687-698$.

[F] R. L. Frank, A remark on eigenvalue estimates and semigroup domination. In preparation.

[FLLS] R. L. Frank, A. Laptev, E. H. Lieb, R. Seiringer, Lieb-Thirring inequalities for Schrödinger operators with complex-valued potentials. Lett. Math. Phys. 77 (2006), 309-316.

[FLS1] R. L. Frank, E. H. Lieb, R. Seiringer, Hardy-Lieb-Thirring inequalities for fractional Schrödinger operators. J. Amer. Math. Soc., to appear.

[FLS2] R. L. Frank, E. H. Lieb, R. Seiringer, Number of bound states of Schrödinger operators with matrixvalued potentials. Lett. Math. Phys., to appear.

[FS] R. L. Frank, R. G. Shterenberg, On the scattering theory of the Laplacian with a periodic boundary condition. II. Additional channels of scattering. Doc. Math. 9 (2004), 57-77.

[H1] D. Hundertmark, On the number of bound states for Schrödinger operators with operator-valued potentials. Ark. Mat. 40 (2002), 73-87.

[H2] D. Hundertmark, Some bound state problems in quantum mechanics. In: Spectral theory and mathematical physics: a Festschrift in honor of Barry Simon's 60th birthday, 463-496, Proc. Sympos. Pure Math. 76, Part 1, Amer. Math. Soc., Providence, RI, 2007.

[HLW] D. Hundertmark, A. Laptev and T. Weidl, New bounds on the Lieb-Thirring constants. Invent. Math., 40 (2000), 693-704.

[L] A. Laptev, Dirichlet and Neumann eigenvalue problems on domains in Euclidean spaces. J. Funct. Anal. 151 (1997), no. 2, 531-545.

[LW1] A. Laptev, T. Weidl, Sharp Lieb-Thirring inequalities in high dimensions. Acta Math. 184 (2000), no. $1,87-111$.

[LW2] A. Laptev, T. Weidl, Recent results on Lieb-Thirring inequalities. Journées "Équations aux Dérivées Partielles" (La Chapelle sur Erdre, 2000), Exp. No. XX, Univ. Nantes, Nantes, 2000.

[LY] P. Li, S-T. Yau, On the Schrödinger equation and the eigenvalue problem. Comm. Math. Phys. 88 (1983), 309-318.

[LL] E. H. Lieb, M. Loss, Analysis. Second edition. Graduate Studies in Mathematics 14, American Mathematical Society, Providence, RI, 2001.

[LSS] E. H. Lieb, H. Siedentop, J. P. Solovej, Relativistic electrons in classical electromagnetic fields. J. Stat. Phys. 89 (1997), 37-59.

[LT] E. H. Lieb, W. Thirring, Inequalities for the moments of the eigenvalues of the Schrödinger Hamiltonian and their relation to Sobolev inequalities. Studies in Mathematical Physics, 269-303. Princeton University Press, Princeton, NJ, 1976.

[P] G. Pólya, On the eigenvalues of vibrating membranes. Proc. London Math. Soc. 11 (1961), $419-433$.

[R] G. V. Rozenblyum, Domination of semigroups and estimates for eigenvalues. St. Petersburg Math. J. 12 (2001), no. 5, 831-845.

[S] B. Simon, Trace ideals and their applications, Second edition, Mathematical Surveys and Monographs 120, American Mathematical Society, Providence, RI, 2005.

Rupert L. Frank, Department of Mathematics, Princeton University, Fine Hall, Princeton, NJ 08544, USA

E-mail address: rlfrank@math.princeton.edu

Ari laptev, Department of Mathematics, Imperial College london, London SW7 2AZ, UK

\& Department of Mathematics, Royal Institute of Technology, 10044 Stockholm, Sweden

E-mail address: a.laptev@imperial.ac.uk \& laptev@math.kth.se 